\title{
Articulación Educación y Trabajo: Un estudio desde la Mirada de los Docentes de la Educación Técnica Regional Chilena y sus Necesidades de Perfeccionamiento Pedagógico
}

\author{
Jorge E. Cid ${ }^{(1)}$, Liliana P. Cuadra ${ }^{(2)}$, Sonia H. Cuevas $^{(3)}$ y Alejandro A. Villalobos ${ }^{(3)}$ \\ (1) Universidad de Concepción, Campus Los Ángeles, Escuela de Educación, Departamento de Ciencias \\ Básicas, Los Angeles-Chile (e-mail: jcid@udec.cl \\ (2) Universidad de Concepción, Campus Concepción, Facultad de Educación, Departamento de Educación \\ Física, Concepción-Chile (e-mail: Icuadra@udec.cl \\ (3) Universidad de Concepción, Campus Concepción, Facultad de Educación, Departamento de Ciencias \\ de la Educación Concepción, Concepción-Chile (e-mail: scuevas@udec.cl; avillalo@udec.cl
}

Recibido May. 26, 2016; Aceptado Jul. 29, 2016; Versión final Sep. 29, 2016, Publicado Feb. 2017

\begin{abstract}
Resumen
En el marco del Convenio de Desempeño UCO1203, entre Ministerio de Educación y Universidad de Concepción (Chile), llamado "Profesores UDEC: Protagonistas del cambio en la sociedad del conocimiento", se realizó un estudio para determinar las necesidades de perfeccionamiento y requerimientos para la formación continua de los docentes de enseñanza media técnico profesional de la región del Biobío. La investigación fue tipo exploratoria, aplicándose una metodología cuantitativa, con diseño de encuesta modelo cuestionario. El universo fue de 116 establecimientos y 3.623 docentes de las cuatro provincias de la región del Biobío en Chile: Concepción, Arauco, Biobío y Ñuble. La muestra aleatoria estuvo constituida por el $80 \%$ de dichos establecimientos, cuyos resultados muestran que los docentes constituyen un grupo homogéneo en motivaciones e intereses profesionales. Destaca en estos intereses comunes, la necesidad de contar con programas de educación continua en las áreas de formación pedagógica, gestión y pasantía.
\end{abstract}

Palabras clave: enseñanza técnico-profesional; trabajo y educación; perfeccionamiento docente; diagnóstico; educación continua

\section{Articulation Education and Work: A Study from the Teachers' Perspective in Chilean Regional Technical Education and their Needs for Educational Improvement}

\begin{abstract}
In the framework of the Performance Agreement UCO2013, between the Ministry of Education and the University of Concepción (Chile), named "UdeC Teachers: Protagonists of change in the society of knowledge", a study has been carried out to determine the needs for improvement and requirements for the continuous formation of technical high-school teachers from four provinces of the Bio-Bio region in Chile: Concepción, Arauco, Biobío and Nuble. The research type was exploratory, applying a quantitative methodology with a questionnaire survey design. The studied universe covered 116 educational centers and 3.623 teachers of all four provinces of the Bio-Bio region. The random sample was made up of $80 \%$ of the already mentioned educational centers, whose teaching staff constituted a homogeneous group with similar professional interests and motivations. Of outstanding relevance among these common interests is the need for continuing education programs in the areas of teacher training management and internship.
\end{abstract}




\section{INTRODUCCION}

Actualmente se requiere ofrecer una formación educativa con destino al sistema productivo. Una evidencia que justifica dicha afirmación es reconocer la aplicación del enfoque de competencias laboral en el quehacer pedagógico de los profesores (Tejada, 2002; Conpes, 2010; Lenoir y Morales-Gómez, 2011). Esta situación es aún más evidente cuando se refiere a la enseñanza técnica profesional, donde los estudiantes requieren de un contacto real y directo con el sistema laboral, lugar donde pueden reconocer y valorar el sentido del trabajo, la importancia del trabajo en equipo, la cultura de la empresa, los procesos de producción y comunicación que caracterizan dicha empresa, entre otras facetas del mundo laboral. De este modo, la institución escolar y particularmente el Liceo Técnico Profesional debe estar preparado para responder a los desafíos del sistema productivo, dando reales oportunidades de colocación a sus egresados. Por cierto, no es solo la institución educativa quien debe ser capaz de responder a este desafío de articulación educación y trabajo, sino que son sus propios profesores los que deben crear la condición de operatividad de dicha asociación.

No hay que olvidar que el enfoque educativo de competencias laborales aplicado al sistema de enseñanza media técnico profesional impone grandes desafíos a los docentes que ejercen en dicho nivel educativo (De Miguel, 2006). Un currículo de orientación laboral se caracteriza por el desafío de desarrollar habilidades, actitudes, valores y normas que permitan a los jóvenes comprender la naturaleza, significado y sentido de la vida laboral; pero también se espera que el estudiante egresado de este nivel sea capaz de desarrollar competencias propias de dicha especialidad laboral, como también lograr una expresión comportamental adecuada a este nivel productivo (Unesco, 2012; Pellegrino y Hilton, 2012). En otras palabras, el establecimiento educacional debe mirar al sistema productivo como el espacio donde sus egresados serán capaces de desarrollar su ejercicio profesional, por lo cual requiere de una permanente actualización, acreditación y certificación institucional. Desafío ingente para sus profesores que deben promover y gestionar el rediseño curricular, los ajustes curriculares y didácticos, la re-evaluación de su perfil, desarrollar competencias genéricas y especificas a su futura labor ocupacional, entre otras tareas docentes que no son fáciles de hacer ni ejecutar.

Todo lo anterior requiere de nuevos docentes y nuevas estrategias de articulación entre el conocimiento y el trabajo, el sistema escolar y el sistema productivo, asociación entre profesores especialistas y trabajadores expertos, cuyas tensiones permite afirmar la necesidad de conocer las opiniones de los profesores de liceos técnico profesional como una primera etapa del fortalecimiento de esta relación estratégica de escuela y trabajo en favor de un desarrollo para todos (Hopenhayn, 2010; Afi Pongo y Obinnim, 2015). El presente artículo expone los resultados de una investigación realizada en el marco del Convenio de Desempeño entre el Ministerio de Educación y la Universidad de Concepción, denominado "Profesores UDEC: Protagonistas del cambio en la sociedad del conocimiento" (CD UCO1203), cuyo objetivo específico fue "Contribuir al mejoramiento de la formación de profesores de educación media técnico profesional de la Región del Biobío". En la construcción del objetivo anterior, se consideraron los diversos cambios en la Enseñanza Media Técnico Profesional (en adelante EMTP) y las nuevas demandas educacionales del sector, asociadas al avance incesante del conocimiento científico-tecnológico, la producción industrialcomercial y el área servicios de la Región del Biobío.

Uno de los ejes del desarrollo estratégico lo constituye la Educación Superior y Técnico Profesional, con un foco especial en Ciencia y Tecnología. La estrategia apunta a atraer una mayor cantidad de estudiantes en diversas áreas definidas como prioritarias en la región, en la cual la característica de especialización sea la vinculación con el mundo productivo laboral y sus requerimientos de innovación, emprendimiento, ciencia y tecnología aplicada. El énfasis se ha puesto en el perfeccionamiento de profesores, cuyo objetivo es desarrollar programas de alto interés y condiciones apropiadas para su participación y aplicación: (a) Educación y formación continua, orientada a la capacitación y la certificación profesional y de oficios, cuyo objetivo es elaborar una política y un plan programático para su desarrollo gradual y aspirar a recursos humanos de alta calidad, empleabilidad, flexibles e innovadores; (b) Mejoramiento de la educación técnica profesional, capacitación y formación dual, cuyo objetivo central es desarrollar una educación técnica profesional y su relación con el sistema productivo regional; y (c) Creación de la carrera universitaria de pedagogía técnico profesional, con el objetivo de mejorar la formación en esta área.

En la educación técnico profesional es posible reconocer algunos antecedentes históricos que ayudan a explicar la actual situación de este sistema educativo. En los últimos 35 años, la creación del Sistema de Educación Superior Chileno en 1981 y la nueva Ley de Universidades, modificó la estructura, funciones y financiamiento de la universidad chilena, lo que obligó a la transformación de algunas universidades tradicionales en derivadas, desapareciendo -entre ellas- la Universidad Técnica del Estado, lo que afectó la formación de profesores técnicos que estaban a cargo de estas importantes funciones y que ninguna otra universidad pública asumió. Recién a finales de la década del 90 y primera década del siglo XXI, recobró 
importancia esta modalidad de enseñanza en el país y en la región. Para ello, se formularon políticas educacionales, planes y normas para formar y perfeccionar profesores TP, las que recién comenzaron a tener vigencia en el año 2001. Así, el Ministerio de Educación postuló al Consejo Superior de Educación una estructura curricular de carácter modular, la que fue asumida por los establecimientos, tanto públicos como privados, de esta rama educacional.

Esta investigación esta contextualizada por el entorno nacional (Sevilla, 2014) e internacional (McCrone et al., 2015) que tensionan estos programas de desarrollo, siendo el punto de partida el diagnóstico provincial de las necesidades de perfeccionamiento de los docentes EMTP como actores de este desarrollo, y de la búsqueda de una nueva articulación entre educación y trabajo.

\section{ANTECEDENTES}

Los antecedentes para abordar esta investigación y que justifican su relevancia investigativa, se resumen en los siguientes puntos: (i) creciente matrícula en establecimientos EMTP; el MINEDUC (2013) indica que cerca de la mitad de la matrícula de Enseñanza Media corresponde a Técnico Profesional; (ii) alto índice de vulnerabilidad de los estudiantes y de los establecimientos; el $61 \%$ de los establecimientos técnico profesionales se encuentran en el decil más vulnerable (MINEDUC, 2009); (iii) falta de investigaciones y de datos empíricos; (iv) dificultad de generar políticas públicas pertinentes que produzcan los impactos esperados (Sepúlveda, 2009); y (v) la cantidad de profesionales universitarios es superior a la de los técnicos de mando medio, donde por cada cuatro ingenieros hay un técnico (Meller y Brunner, 2009). Del mismo modo, los estudios de naturaleza exploratoria y/o los diagnósticos sobre los requerimientos de perfeccionamiento de los docentes EMTP son poco frecuentes, tanto a nivel regional como nacional, a pesar de la urgente necesidad de formación y capacitación técnico - pedagógica para contribuir al mejoramiento del aprendizaje de los estudiantes. Esta argumentación se fortalece cuando el MINEDUC (2010) plantea que sólo el $52 \%$ de los docentes que imparten clases en la EMTP posee el título de profesor.

Arancibia et al. (2008) sintetizan esta problemática en los siguientes aspectos: (1) déficit real de docentes con formación pedagógica adecuada para conducir los procesos de enseñanza-aprendizaje técnico; (2) inexistencia de una oferta educativa de formación flexible para responder adecuada y eficazmente a las necesidades de tan heterogéneos participantes; (3) los docentes técnicos deberían estar en condiciones de facilitar a sus estudiantes el ingreso y permanencia en el mundo laboral; (4) el perfeccionamiento docente debería integrar variedad de situaciones o ambientes de aprendizajes que permitan atender las diferencias individuales; y (5) se necesita aplicar en los procesos de formación y actualización profesional docente, los códigos de la modernidad, especialmente las referidas a las nuevas TICs. El estudio de Sepúlveda et al (2009) aporta interesantes elementos respecto a los actores de la EMTP. En oposición a lo anterior, los diagnósticos negativos que se han presentado durante los últimos años, contrastan fuertemente con la opinión favorable respecto de la EMTP por parte de estudiantes y profesores. En el caso de los estudiantes, los autores plantean que el producto objetivable (título técnico de nivel medio) es valorado como un capital cultural y como un medio para continuar trayectorias educativas en la Educación Superior, así como oportunidades laborales y de empleo.

En el año 2007, los programas fueron evaluados por el CIDE con la participación de 1157 docentes, de los cuales $132(11,41 \%)$ impartía clases en la región del Biobío. Los resultados evidenciaron serias deficiencias en la programación de metas fijadas por el CPEIP, puesto que solo se cumplió en un 57\% en el primer año. En efecto, a nivel nacional, los programas alcanzaron una cobertura del $7.5 \%$ de la población objetivo. A su vez, la encuesta de satisfacción de los docentes participantes muestra una evaluación positiva en gestión de la información, postulación, satisfacción y pertinencia del programa. Respecto a la motivación para participar en los cursos, un 29.9\% expresó motivaciones económicas, mientras que el 91,1\% declaró el deseo de establecer nuevos vínculos y experiencias, y un $89 \%$ declaró querer ser mejor profesor o profesora.

Posteriormente se realizó una evaluación de seguimiento a los postítulos en pedagogía para docentes de la formación diferenciada de EMTP (2008-2009). Las principales inclinaciones para participar fueron: hacer bien el trabajo (54,4\%), cumplimiento de la normativa para seguir trabajando (19,1\%), beneficio beca $(43,4 \%)$ y la confianza que entrega la Universidad $(28,7 \%)$. En este contexto las tasas de retiro, deserción y reprobación fueron escasas. Luego de la realización del curso, tres de cada cuatro participantes reporta un alto o muy alto nivel de satisfacción con el postítulo. Capablanca Consultores (2008) concluyen que la evaluación sobre la oferta de postítulos destaca coherencia interna y equilibrio, entre la formación teórica y práctica; así mismo, entre el diseño y la práctica observada en terreno. En la evaluación de los programas de perfeccionamiento para profesores, sumado a la inexistencia de una política de Estado en la formación continua, determinan falta de objetivos sistemáticos y coherentes, además de carencia en coordinación con otras políticas educacionales vinculadas a la mejora del nivel de aprendizaje de los estudiantes. Donoso 
(2008) señala que el MINEDUC estableció una serie de programas focalizados, heterogéneos en cuanto a su duración, intensidad y modalidad; por consecuencia, es un "área de carácter desregulado", ya que sólo una parte de ellos está bajo la supervisión del CPEIP, sumado a una falta de ajuste respecto a los diagnósticos del sector. Ávalos (2002) indica que falta mayor coordinación en la planificación del perfeccionamiento, puesto que -según las necesidades- las acciones nuevas pueden reemplazar a las antiguas, sin considerar las ya existentes. Como elementos positivos, se destaca la mejor actitud de los docentes para el desempeño en el aula, el apoyo al desarrollo profesional y el aprendizaje colaborativo. Para Beca (2006) uno de los desafíos de la formación continua es articular la formación inicial en las universidades con la formación en servicio promoviendo la coherencia de los enfoques, reforzando así las oportunidades de capacitación a docentes de comunidades apartadas y zonas rurales.

Arancibia et al. (2008), en relación a la formación continua, señalan que las necesidades formativas mayormente demandadas son tres: (i) Dimensión Pedagógica: la gestión escuela-mundo productivo, el uso de TICs y la evaluación de los aprendizajes; (ii) Dimensión Disciplinar: la preparación para el mundo laboral, proyectos interdisciplinarios vinculantes y demandas laborales del sector productivo; y (iii) Dimensión Transversal: liderazgo, ética profesional y formación ciudadana. Los investigadores señalan a partir de las evidencias, la desarticulación entre las propuestas curriculares de formación docente y el perfil profesional diseñado para el mercado laboral.

Calisto et al. (2010), en la investigación sobre "Ofertas flexibles de la Universidad de Concepción para la formación de Profesores Técnicos" realizada entre esta universidad y Chilecalifica, elaboraron un perfil de egreso para profesores de EMTP basado en competencias múltiples validado por panel de expertos y especialistas nacionales y regionales en el área, el que se consolidó en la ejecución del Programa de Formación Docentes Técnicos. Por otra parte las Bases curriculares para la Enseñanza Media Técnico Profesional entregadas por el MINEDUC (2013), establecen cambios desde el punto de vista metodológico, porque se adecúan a las nuevas corrientes en educación y también en lo estructural, puesto que se plantean cambios de especialidades, esto hace que los profesores deban perfeccionarse acorde con estos nuevos lineamientos.

El objetivo general de la investigación fue diagnosticar las necesidades y requerimientos del perfeccionamiento y formación continua de los docentes de Educación Media Técnico Profesional de cada una de las cuatro provincias de la Región del Biobío. Para su cumplimiento se formularon los siguientes objetivos específicos: (1) Caracterizar a los docentes actualmente en servicio en los establecimientos de Enseñanza Media Técnico Profesional de cada una de las provincias de la región; (2) Diagnosticar las necesidades y requerimientos de perfeccionamiento docente a partir de sus opiniones y percepciones para el logro de competencias de gestión y de aprendizaje; (3) Determinar los requerimientos de formación continua contextualizada en su realidad profesional; y (4) Determinar la percepción valórica que asignan los profesores a las pasantías técnicas realizadas fuera de sus establecimientos.

\section{METODOLOGIA}

La investigación fue de tipo exploratoria - descriptiva, aplicándose una metodología cuantitativa con diseño de encuesta modelo cuestionario; su diseño es no experimental, ya que no existe control de las variables por parte de los investigadores. El artículo se enfoca especialmente en exponer los resultados obtenidos de la investigación realizada en la Región del Biobío, en relación a los objetivos que se refieren especialmente al diagnóstico de las necesidades de perfeccionamiento docente y determinación de los requerimientos para la formación continua.

\section{Universo y muestra}

El universo de los establecimientos de EMTP de la Región del Biobío está constituido por 116 unidades en las cuatro provincias de la región mencionada (Concepción, Ñuble, Biobío y Arauco) y por 3.623 docentes que laboran en dichas instituciones.

La muestra correspondió al 83\% del universo, como criterio establecido en el Convenio de Desempeño UCO 1203, llegando a 96 unidades educativas y 424 docentes. El método de muestreo es probabilístico, bietápico, de conglomerados y estratificado. La selección de la muestra tuvo las siguientes dos etapas: (1) En la primera etapa se definió la unidad primaria de muestreo, correspondiendo la selección de las unidades educativas de EMTP, a través de números rándom; y (2) La siguiente etapa fue la selección de docentes dentro de las unidades educativas, la que también se realizo mediante números rándom.

La selección de los establecimientos se realizó a través de un muestreo estratificado, el cual se utilizó la tipología clasificatoria de establecimientos de EMTP del Ministerio de Educación de Chile (MINEDUC). 


\section{Instrumento para la recolección de información}

En función de los objetivos del estudio, se diseñó un cuestionario de 36 preguntas cerradas. El proceso de validación del instrumento se realizó en dos etapas, donde la primera fue a través de juicio de expertos y la segunda mediante una prueba piloto. El instrumento consta de cuatro dimensiones, según los objetivos: (i) Caracterización socio-demográfica y laboral de los docentes; (ii) Perfeccionamiento realizado; (iii) Necesidades percibidas de perfeccionamiento; y (iv) Evaluación del perfeccionamiento realizado mediante pasantías. La aplicación en terreno del instrumento lo realizó el equipo de encuestaje en forma presencial, en cada uno de los establecimientos donde los docentes de la muestra trabajan en sus respectivas provincias.

\section{RESULTADOS Y DISCUSIÓN}

En primer lugar, se realizó un análisis descriptivo de los datos y luego uno de carácter inferencial, que incluyó las pruebas Chi-Cuadrado, Correlación de Pearson y de Sperman, las que entregan resultados estadísticamente significativos. Finalmente, se destaca que para cada una de las pruebas de Chi-Cuadrado se utilizó la Prueba de Corrección de Yates, cuyo fin es controlar bajos efectivos en tablas de dos por dos; estos son datos ausentes por diversas causas: no responde, no sabía, no aplica, no es pertinente, entre otros.

\section{Análisis descriptivo regional}

La distribución por sexo de la muestra es homogénea. En efecto un $51,4 \%$ de los docentes son mujeres, mientras que el $48,6 \%$ son hombres. Respecto a la edad de los encuestados la media es 41,39 años, con un rango de 54 años, siendo la máxima 76 años y la mínima 22 años. La moda de este conjunto de datos es de 32 años, por lo cual se presume que los docentes encuestados ya cuentan con cierto nivel de experiencia laboral. Los docentes con menos de un año de trabajo, corresponde al 12,5\%, de 1 a 5 años el $31,1 \%$. Entre 6 y 10 años, un 19,1\% de los profesores señala tener esta antigüedad. Entre 11 y 15 años, el $12,3 \%$ y entre 16 y 20 años de antigüedad, un 7,6\%. Finalmente con más de 20 años un 17,4\%. Con respecto a la modalidad de los establecimientos donde trabajan los encuestados, se aprecian dos grandes grupos: un $69,5 \%$ de los sujetos trabaja en establecimientos Técnico Profesionales y el $30,5 \%$ de docentes trabaja en Centros Educativos Polivalentes.

En relación al plan de estudios donde se desempeñan los docentes, el 52,6\% indica realizar su trabajo en el Plan Común, seguido del 27,1\% en el Plan de Especialidades. Finalmente el $20 \%$ de los profesores encuestados menciona desarrollar su labor en ambos Planes. El 67,3\% de los encuestados declara estar en posesión del título de profesor. A esto le sigue la categoría Otro un 19,7\% y finalmente se ubican con el titulo Técnico de Nivel Superior un 7,8\% y Técnico Nivel Medio un 5,2\%. Se debe destacar que en esta pregunta, los sujetos podían responder más de una opción, por lo cual se debe asumir que un mismo individuo puede estar en posesión de más de un título profesional.

Los encuestados sin título de profesor y que cuentan con habilitación docente que proporciona el MINEDUC, es del $72,2 \%$. El $27,8 \%$ declara no poseer la habilitación ministerial para realizar clases, lo cual indica que aproximadamente 1 de cada 4 profesionales no docentes que trabaja en Liceos Técnico Profesionales imparte asignaturas sin la debida autorización de la autoridad competente. De los profesionales que no poseen título universitario de profesor, un $85,2 \%$ declara su deseo de obtener dicho título, es decir, se observa disposición de los sujetos encuestados para cursar carreras relacionadas con la pedagogía. En contraste, un $14,8 \%$ de los encuestados declara no sentirse interesado en obtener el título de profesor.

\section{Diagnóstico del perfeccionamiento realizado}

En relación a la capacitación realizada, el $66,7 \%$ de los docentes afirma haber realizado perfeccionamiento en los últimos 5 años, en contraste con el 33,3\% de los docentes que indica no haber realizado ningún tipo de capacitación en este periodo. Además, el $23 \%$ menciona poseer capacitación en Orientación Educativa; el $19,2 \%$ de los encuestados declara tener especialización en Aprendizaje Basado en Competencias, siguiéndole el $16,1 \%$ y $15 \%$ con Evaluación por Competencias y Planificación de Clases. Finalmente el $14,5 \%$ de los docentes realizó perfeccionamiento en Didáctica para el Aprendizaje y un $12,3 \%$ en Emprendimiento. En cuanto al área de Gestión Escolar, el 20,4\% de los docentes indica haber hecho perfeccionamiento en Liderazgo Escolar, seguido de Gestión Escolar, Administración Educacional y Uso de TIC's para gestión Pedagógica, cada de ellas con el 19,5\% y en Supervisión Educacional sólo un 3,5\%. 
Necesidades percibidas de perfeccionamiento.

En las Percepciones del perfeccionamiento realizado, los mayores porcentajes de "muy de acuerdo" y "de acuerdo" se concentran en las opciones: a) Contribución al mejoramiento del aprendizaje (85.4\%), b) Contribución al mejoramiento de la docencia $(88.8 \%)$, c) Integración óptima entre la teoría y la práctica (78.6\%) y d) Aplicación de los conocimientos al trabajo de aula (67\%). En cambio, los niveles "muy en desacuerdo" y "en desacuerdo" son: a) El perfeccionamiento realizado habilita para trabajar con estudiantes vulnerables, $(19,7 \%)$ y b). El perfeccionamiento contribuye a mejorar las oportunidades laborales (19\%).

Necesidad de realizar un postítulo en los próximos dos años: El 41,1\% de los docentes declara estar "muy de acuerdo" con esta afirmación, mientras que el 28,6\%, está "de acuerdo". "En Desacuerdo" ó "Muy en Desacuerdo" suman el 10,2\%.

Interés para realizar cursos de perfeccionamiento: Respecto del interés de los encuestados por realizar cursos de perfeccionamiento, se tomaron datos que apuntaban a dos dimensiones: formación pedagógica y gestión escolar. En la primera dimensión los temas de mayor interés son: Emprendimiento, Orientación educativa y Uso de TIC's para el aprendizaje. Por el contrario, los de menor interés fueron Planificación, Evaluación de los aprendizajes y Didáctica para el aprendizaje. En relación a la segunda dimensión los de mayor interés fueron Formación de Directores de Excelencia, Supervisión Educacional, Uso de TIC's para Gestión Pedagógica, y con un interés menor Administración Educacional, Planificación Educacional, Gestión y Liderazgo Escolar.

Motivaciones para el Perfeccionamiento: Se observa que un $98,1 \%$ de los encuestados está Muy de Acuerdo y de Acuerdo con Mejorar los Aprendizajes de los estudiantes, mientras el 97,4\% con Mejorar el Desempeño del Establecimiento. En cambio, Obtener Mejor Remuneración presenta una mayor dispersión que se observa en los siguientes porcentajes: Muy de Acuerdo y de Acuerdo 37,9\%, Ni Acuerdo, Ni en Desacuerdo 35,7\%, y En Desacuerdo y Muy en Desacuerdo, con un 26\%.

\section{Evaluación del perfeccionamiento realizado mediante pasantías}

Evaluación Modalidad Pasantía: Los encuestados evalúan con un promedio 5,45 la modalidad de pasantía, y con una desviación típica de 1,5 puntos, lo que se explica dados los valores mínimo 1 y máximo 7 de la evaluación. La media aritmética es favorable, ya que está solo en 1,55 puntos bajo el valor máximo.

Pasantías realizadas fuera del Establecimiento durante los últimos 5 años: El 88,9\% de los docentes señala no haber realizado pasantías fuera de su establecimiento durante los últimos cinco años. En contraste, sólo el $11,1 \%$ respondió afirmativamente. Se deduce, por lo tanto, que la modalidad de pasantías, no ha sido utilizada por los sujetos encuestados o bien, no ha sido implementada por los establecimientos en los que ellos trabajan.

Evaluación de las pasantías realizadas: La evaluación es positiva, con una media de 6,02 y con una dispersión de 1,33 de un rango de 1 a 7 .Sumado a lo anterior, se destaca la evaluación favorable que realizan los docentes del perfeccionamiento realizado fuera del establecimiento.

Impacto de la pasantía en la formación profesional: El $80 \%$ de los encuestados menciona que ha sido positivo y que además, ha tenido posibilidades de aplicar lo aprendido en el aula. Sin embargo, un 18,6\% indica que si bien ha sido positivo, no ha tenido oportunidad de aplicar lo aprendido. Finalmente, sólo un $2,3 \%$ de los docentes que ha realizado una pasantía la evalúa negativamente.

\section{Análisis inferencial}

En esta sección se realizan pruebas de hipótesis bi-variadas con el fin de encontrar asociaciones entre las variables que se recogen del estudio. Lo primero que se realiza es establecer asociación entre: 1) Provincia y título de profesor (chi-cuadrado); (2) Provincia y habilitación docente (chi-cuadrado); (3) Provincia y realización de cursos de perfeccionamiento (chi-cuadrado); (4) Provincia y contribución de los cursos de perfeccionamiento (chi-cuadrado); (5) Provincia e interés por participar en cursos de perfeccionamiento (ANOVA); (6) Provincia y evaluación sobre modalidad y pasantías desarrolladas (ANOVA); (7) Posesión de título de profesor y realización de cursos de perfeccionamiento (chi-cuadrado); (8) Posesión de habilitación docente y realización de cursos de perfeccionamiento (chi-cuadrado) (9) Edad y realización de cursos de perfeccionamiento ( $T$ de Student); y (10)Edad e interés en participar de cursos de perfeccionamiento (correlación de Pearson). 
El principal factor de distinción para el presente trabajo es la variable "Provincia", el análisis en general arrojó sólo asociaciones no significativas en los distintos test realizados. La única asociación significativa que distingue entre provincias es la que las vincula con la evaluación que hacen los docentes a la modalidad de pasantía.

Tabla 1: Test ANOVA, evaluación de 1 a 7 hacia la modalidad de pasantías, según provincia $(\mathrm{Sig}=0,000063)$.

\begin{tabular}{|l|c|c|c|}
\hline & $N$ & Media & Desviación estándar \\
\hline Concepción & 137 & 5,61 & 1,421 \\
\hline Ñuble & 117 & 5,79 & 1,181 \\
\hline Biobío & 82 & 4,8 & 2,105 \\
\hline Arauco & 29 & 5,14 & 1,457 \\
\hline Total & 365 & 5,45 & 1,58 \\
\hline
\end{tabular}

Según la Tabla № 1, hay una evaluación más positiva entre los docentes que trabajan en la provincia de Ñuble $(5,79)$ seguida de la de los docentes de la provincia de Concepción $(5,61)$, concentrando ambas un subconjunto homogéneo, según los análisis realizados. Los docentes de Arauco $(5,14)$ y Biobío $(4,8)$ se distinguen significativamente de la evaluación hecha al grupo anterior. Sin embargo, esto no se traduce en una evaluación estadísticamente diferente entre provincias para la evaluación de pasantías realizadas, que arrojan un empate estadístico entre los promedios de las cuatro provincias. Al centrar el análisis sobre la realización de perfeccionamiento, no existe relación entre rama técnica del establecimiento donde trabaja el docente y la realización de cursos de perfeccionamiento en los últimos 5 años $\left(x^{2}=2,34 ; p=0,8\right)$. Tampoco existe relación entre tener título de profesor de nivel universitario y la realización de dichos cursos $\left(x^{2}=0,119 . p=0,73\right)$.

Tabla 2: Profesor o Pedagogo Titulado, según realización de perfeccionamiento en Aprendizaje basado en competencias $\left(X^{2}=9,46\right.$; Sig. $\left.=0,002\right)$.

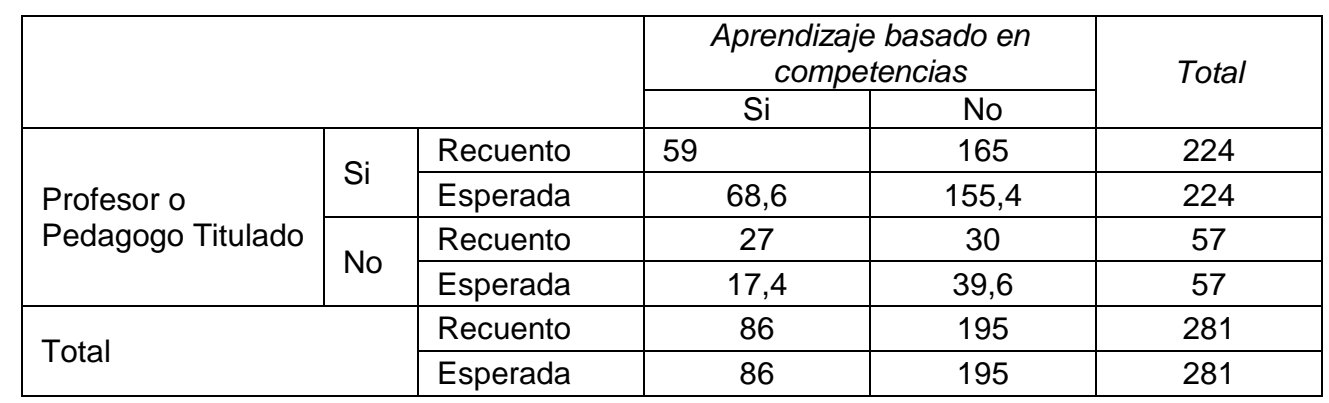

Existe una relación significativa entre la posesión de título de pedagogo universitario y el haber realizado perfeccionamiento sobre Aprendizaje Basado en Competencias. Los docentes que más se capacitan en esta área son aquellos que no tienen título de profesor o pedagogo universitario. Esta es la única relación existente entre los diferentes perfeccionamientos consultados y la posesión de dicho título, resultando valores no significativos en el resto de los cursos consultados.

Tabla 3: Posesión de habilitación docente autorizada por MINEDUC, según haya realizado perfeccionamiento en Didáctica para el aprendizaje $\left(X^{2}=4,986\right.$; Sig.=0,026).

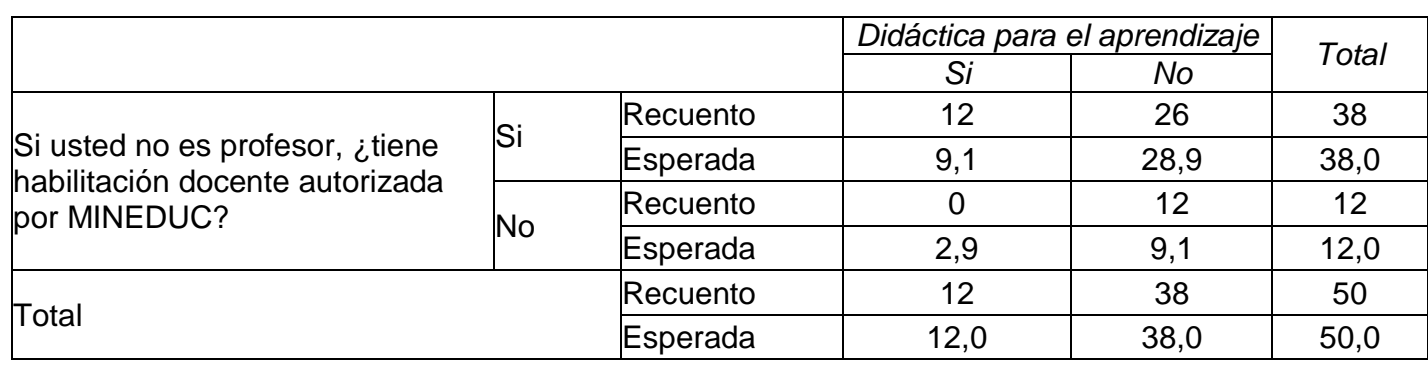

Según la tabla 3 , no existe una vinculación significativa entre la realización de cursos de perfeccionamiento y la habilitación docente entregada por MINEDUC $(x 2=0,605 ; p=0,437)$. A pesar de ello, existe una relación significativa entre el tener habilitación docente y el haber realizado cursos de Didáctica para el Aprendizaje. Sin embargo, los datos muestran que quienes tienen la habilitación docente son quienes han hecho mayoritariamente estos cursos, mientras que quienes no la poseen, dicen haber realizado menor proporción 
de cursos de perfeccionamiento en esta área. Estos datos deben tomarse con cuidado, puesto que en primer lugar se observan bajos efectivos en una de las casillas y, además, la cantidad total de casos es pequeña, lo que en la prueba de chi cuadrado es relevante, ya que el tamaño afecta la significancia de la dependencia de variables. Al revisar la vinculación entre la edad de los docentes y el interés por realizar cursos de perfeccionamiento, se hallaron algunas relaciones significativas que se presentan en la tabla 4.

Tabla 4: Correlaciones de Pearson, Edad e Interés por participar en un curso de perfeccionamiento ( ${ }^{*}$ Todas las correlaciones significativas al 0,05. Se eliminaron las relaciones no significantes en la tabla).

\begin{tabular}{|c|l|c|}
\hline \multirow{2}{*}{ Criterio } & \multicolumn{1}{|c|}{ Cursos de Perfeccionamiento } & Coef. Correlación* \\
\hline \multirow{3}{*}{ Formación Pedagógica } & Enseñanza Basada en Competencias Múltiples & $-0,13$ \\
\cline { 2 - 3 } & Didáctica para el Aprendizaje & 0,275 \\
\cline { 2 - 3 } & Uso de TIC's para el Aprendizaje & $-0,126$ \\
\hline \multirow{3}{*}{ Gestión educativa } & Orientación Educativa & 0,103 \\
\cline { 2 - 3 } & Formación de Directores de Excelencia & 0,117 \\
\cline { 2 - 3 } & Uso de TIC's para la Gestión Pedagógica & $-0,159$ \\
\hline
\end{tabular}

De la Tabla 4 se puede inferir que los profesores más jóvenes tienden a preferir participar en cursos de perfeccionamiento de Enseñanza Basada en Competencias Múltiples ( $r=-0,13)$, en el de Uso de TIC's Orientado hacia el Aprendizaje $(r=-0,126)$ y en el de Uso de TIC's para Gestión Pedagógica $(r=-0,159)$. En tanto, en la medida que la edad de los profesores aumenta, se incrementa el interés por participar en cursos de Didáctica para el Aprendizaje $(r=0,275)$, de Orientación Educativa $(r=0,103)$ y de Formación de Directores de Excelencia $(r=0,117)$.

\section{Síntesis e interpretación de los datos}

Tal vez, sea necesario ofrecer una síntesis de los principales hallazgos de los datos obtenidos, con el propósito de facilitar la comparación de ideas y una eventual discusión de los resultados planteado en este trabajo. De este modo, al ser consultados acerca del perfeccionamiento realizado por los profesores que enseñan en los establecimientos técnicos de las cuatro provincias de la Región del Biobío, dos tercios de la muestra de ellos indican haber realizado alguno en los últimos 5 años. Asimismo, un 67,2\% de los encuestados mencionan la necesidad de cursar un perfeccionamiento en los próximos dos años, lo que podría ser tomado como indicador de los requerimientos de los docentes, de los ajustes y toma de decisiones que deben considerar los diferentes niveles de estudios universitarios que imparten dicho perfeccionamiento.

En relación al interés que manifiestan los profesores de las cuatro provincias sobre el perfeccionamiento vinculado a la Formación Pedagógica, destacan particularmente las áreas de Orientación, Aprendizaje Basado en Competencias y el Uso de TIC's para el Aprendizaje; por su parte, en aquellos vinculados a la Gestión Escolar se destaca Liderazgo y Gestión Escolar y Uso de TIC's para la Gestión Pedagógica. Los profesores más jóvenes, por su parte, están más interesados en perfeccionarse en áreas vinculadas a temas relativamente emergentes, como uso de TIC's y de Aprendizaje de Competencias. EI uso de TIC's, de hecho, se observa como una creciente necesidad tanto a nivel estrictamente pedagógico como de gestión educativa. El desarrollo tecnológico y los requerimientos de estudiantes cada vez más cercanos a las tecnologías de información y la sensación de desconocimiento e incertidumbre ante innovaciones constantes, puede explicar esta extendida necesidad de los docentes para ser capacitados en dicha área, lo que también les puede permitir un ascenso a cargos directivos.

Vinculado a lo anterior, los profesores técnicos con mayor experiencia de las cuatro provincias se interesan en temas de Gestión y Didáctica Centrada en el Aprendizaje. Así, se propone que los profesores con inclinación hacia la Gestión Directiva se alejan de la docencia directa en el aula. Los docentes motivados por la Didáctica, en cambio, pretenden mejorar sus prácticas ante las nuevas demandas pedagógicas derivadas de los cambios curriculares y generacionales. Esto sería consistente con la declaración que tienen estos últimos docentes que, en su mayoría, aducen como principal motivo para capacitarse, la necesidad de realizar mejoras en el aprendizaje de sus estudiantes en los liceos técnicos provinciales.

La valoración que realizan los profesores técnicos de las distintas provincias sobre el perfeccionamiento ya realizado es mayoritariamente positiva, indicando que efectivamente ha sido útil para su trabajo en el aula y en su relación con los estudiantes. El único indicador que tiene una evaluación crítica, está referido a las expectativas de ascenso laboral que han tenido posterior al perfeccionamiento. 
En relación a la importancia otorgada a las pasantías y sus modalidades de realización, se ha constatado que los profesores técnicos que enseñan en liceos técnicos de las provincias de la Región del Biobío, manifiestan que éstas tienen una utilidad efectiva en su trabajo docente. Esto se reafirma con la alta tasa de respuesta sobre la aplicación en el aula que tiene la pasantía para quienes la han realizado. También se verifica el hecho de haber realizado una pasantía permite tener una mejor opinión acerca de esta modalidad de perfeccionamiento. Finalmente, cabe señalar que, a partir de los análisis antes expuestos, no se observan diferencias significativas en las respuestas de los docentes que enseñan en los diferentes establecimientos técnicos de la enseñanza media de las cuatro provincias de la Región del Biobío, en relación a los niveles y tipos de experiencia, formación y lugares de trabajo.

En suma, de los datos obtenidos se deducen que los resultados reflejan que los docentes de las cuatro provincias de la Región del Biobío componen un grupo homogéneo con motivaciones e intereses profesionales muy similares en el sistema educación técnica profesional. Situación que valida la elección metodológica del presente estudio de caso; cuyo procedimiento puede ayudar a su réplica o aplicación en otros contextos socio culturales, sean naciones o internacionales.

\section{DISCUSION FINAL}

La temática abordada en el presente estudio permite afirmar que el sistema de la educación técnico profesional es un área que puede ofrecer una efectiva vinculación entre el mundo escolar y el mundo productivo, pero que requiere de una real asociación de estos ámbitos, donde los profesores pueden ser valiosos agentes que permitan promover la incorporación del conocimiento como valor agregado al sistema productivo. Al centralizar en la figura del profesor de la enseñanza media técnica profesional se ha procurado destacar su rol y función didáctica y pedagógica en la promoción de un aprendizaje de calidad y, a la vez, la oportunidad de ofrecer una formación integral en consonancia con la exigencia del mercado laboral regional.

Un segundo aspecto que se puede enfatizar es la oportunidad de hacer una llamada de atención a la opinión pública y a sus autoridades para promover una política pública de fortalecimiento de este sector educativo que atiende a los alumnos más vulnerables y desposeídos de la sociedad chilena, como un mecanismo efectivo de un mejor desarrollo humano y laboral a sus estudiantes egresados. De igual modo se requiere diseñar una política de perfeccionamiento a los profesores de dicho sector, que incluya postítulos de formación pedagógica especializada, cursos de capacitación y pasantías, lo que permitirá un mejor acercamiento entre el mundo escolar y el productivo. Por último, frente a los aspectos metodológicos y epistemológicos que subyacen en la ejecución del presente estudio, cabe señalar que son adecuados para la comprensión de la problemática abordada. Situación que ha querido reflejar el presente artículo.

\section{CONCLUSIONES}

De acuerdo a los resultados, se pueden extraer las siguientes conclusiones principales:

1) el sistema de la educación técnico profesional es un área que puede ofrecer una efectiva vinculación entre el mundo escolar y el mundo productivo,

2) se requiere diseñar una política de perfeccionamiento a los profesores de dicho sector, que incluya postítulos de formación pedagógica especializada, cursos de capacitación y pasantías,

3) la comprensión de la problemática abordada se fundamentó en aspectos metodológicos y epistemológicos adecuados, que subyacen en la ejecución de este estudio, lo que se ha querido reflejar en el presente artículo.

\section{AGRADECIMIENTOS}

Proyecto financiado por el Ministerio de Educación de Chile y la Universidad de Concepción: CD-UCO 1203 "Profesores UDEC: Protagonistas del cambio en la sociedad del conocimiento".

\section{REFERENCIAS}

Afi Pongo, N. y Obinnim, E. Changing Landscape of Industry Practice: The Role of Quality Technical Vocational Education and Training in Ghana, http://www.iiste.org/Journals/index.php/ADS/article/view/23707/24278; ISSN (Online): 2225-0603, Arts and Design Studies, vol.33, 1-9 (2015)

Arancibia, M.; Miranda, C.; Pérez, H.; Koch, T. Necesidades de formación permanente de docentes técnicos, doi: S0718-07052008000100001, Estudios Pedagógicos. 34 (1), 7-26 (2008) 
Ávalos, B. La formación docente continúa en Chile. Desarrollo, logros, limitaciones. En: Formación docente; un aporte a la discusión. La experiencia de algunos países. UNESCO/OREALC, Santiago, Chile (2002)

Beca C. E. Las Políticas de Formación Continua de Docentes. En Arellano M. y Cerda A. M. (editoras), Formación Continua de Docentes: un camino para compartir. CPEIP, Santiago (2006)

Calisto, R. et al. Ofertas flexibles de la Universidad de Concepción para la formación de Profesores Técnicos. Universidad de Concepción y Chilecalifica, Dirección Docencia, Universidad de Concepción (2010)

Capablanca Consultores. Evaluación en profundidad de los programas de perfeccionamiento docente para profesionales de la educación. Ministerio de Educación. Informe Final. Institución evaluadora: Cartes y LeBert Limitada. Santiago, Chile (2008)

CIDE. Estudio sobre la implementación curricular en la Enseñanza media Técnico-profesional. Informe final. Santiago, Chile (2009)

CONPES. Lineamientos de política para el fortalecimiento del sistema de formación de capital humano SFCH (3674). Recuperado de http://www.colombiaaprende.edu.com/html/home/1592/articles277170_3674.pdf (2010)

De Miguel, M. Modalidades de enseñanza centradas en el desarrollo de competencias orientaciones para promover el cambio metodológico en el espacio europeo de educación superior. Recuperado de http://www.uvic.es/sites/default/files/ensenanza_para_competencia.PDF (2006)

Donoso, S. El perfeccionamiento docente en Chile (1990-2007): ¿Estado versus mercado?. Doi: S141324782008000300003. Revista brasileira de Educação, 13(39), 437-454, (2008)

Hopenhayn, M. Repensar el trabajo. Buenos Aires, Argentina: Grupo Editorial Norma (2001)

Lenoir, Y. y Morales-Gómez, M. A. El Enfoque por Competencias y Profesionalización de la Enseñanza: Una Clarificación Conceptual. http://www.rinace.net/reice/numeros/arts/vol9num1/art3.pdf; ISSN: 1696-4713, REICE. Revista Iberoamericana sobre Calidad, Eficacia y Cambio en Educación, 9 (1), 46-64 (2011)

McCrone, T., O'Beirne, C., Sims, D. y Taylor, A. A Review of Technical Education. Slough: England, National Foundation for Educational Research (2015)

Meller, P.; Brunner, J.J. Educación Técnico Profesional y Mercado Laboral en Chile: Un reader. Gobierno de Chile, UDP. (CPCE). Universidad de Chile (Ingeniería Industrial), Santiago de Chile (2009)

MINEDUC. Estado y perspectivas de la enseñanza media técnico profesional en Chile: un estudio sobre las orientaciones estratégicas predominantes en los actores. FONIDE. Santiago, Chile (2009)

MINEDUC. Nuevas Bases Curriculares de Séptimo a Segundo Medio y Programas de Estudio TP, aprobado por el Consejo Nacional de Educación. Unidad de Currículo. Santiago, Chile (2013)

MINEDUC. Serie Evidencias: Radiografía de la Educación Técnico Profesional en Chile. Centro de Estudios. Santiago, Chile (2013)

Pellegrino, J.W. y Hilton, M.L. (Eds.). Education for Life and Work: Developing Transferable Knowledge and Skills in the 21st Century. DOI: 10.17226/1339 (2012)

Sepúlveda, L.; Ugalde, P.; Campos, F. Estado y perspectivas de la enseñanza media técnico-profesional en Chile: un estudio sobre las orientaciones estratégicas predominantes en los actores. FONIDE, CIDE, Universidad Alberto Hurtado, Santiago, Chile (2009).

Sevilla, P. La educación técnica en Chile y Estados Unidos desde una perspectiva histórica y comparada, doi S0718-45652014000100010, Revista Calidad en la Educación, (40), 297-317 (2014)

Tejada, J., El docente universitario ante los nuevos escenarios: implicaciones para la innovación docente, http://www.saber.ula.ve/accionpe/; ISSN: 1315-401X, Rev. Acción Pedagógica, 11(2), 30-42 (2002)

UNESCO. Consenso de Shanghái recomendaciones del Tercer Congreso Internacional sobre Educación y Formación Técnica y Profesional "transformar la EFTP: forjar competencias para el trabajo y la vida" (ED2012/WS/9). Recuperado de http://unesdoc.unesco.org/images/0021/002176/217683s.pdf (2012) 